\title{
Cardiovascular-metabolic responses and oxygen uptake kinetics during kayak paddling in normobaric hypoxia in young flatwater kayakers
}

Authors' Contribution: A Study Design

B Data Collection

C Statistical Analysis

D Data Interpretation

E Manuscript Preparation

F Literature Search

G Funds Collection

\author{
Elvin Onarici Güngör ${ }^{A B C D E F}$, Celil Kaçoğlu ${ }^{A B C D E F}$, Ali Onur CerraheF, illker Yılmaz ${ }^{A E F}$ \\ Eskişehir Technical University, Faculty of Sport Sciences, Eskişehir, Turkey
}

abstract

Background: The aim of the study was to evaluate response of cardiovascular, metabolic and oxygen consumption kinetics at kayak paddling exercise in the normoxic and normobaric environment.

Material and methods: Seven kayakers (age: $16 \pm 1.2$ year; height: $165 \pm 8.3 \mathrm{~cm}$; body weight: $53 \pm 8 \mathrm{~kg}$; fat percentage: $17 \pm 4.4 \%$; $\mathrm{VO}_{2} \max : 34.57 \pm 9.24 \mathrm{ml} . \mathrm{kg}^{-1} . \mathrm{dk}-1$; Maximal La: $8.72 \pm 2.75 \mathrm{mM}$ ) with four years' experience participated in the study. All subjects performed incremental maximal and sub-threshold constant load kayak paddling tests in normoxic and normobaric hypoxic conditions on different days. According to maximal tests results, the individual anaerobic threshold and $\mathrm{VO}_{2}$ max values were determined.

Results: $\quad \mathrm{VO}_{2} \max , \mathrm{HRmax}$, peak power and maximal lactate values demonstrated statistical significant differences between the normobaric hypoxia and normoxic environment. The response of $\mathrm{VO}_{2}$ max was found higher in the normoxic than the normobaric environment. Oxygen uptake kinetic was statistically $(p<0.05)$ faster, and $\mathrm{O}_{2}$ values were lower $(\mathrm{p}<0.05)$ in the normoxic than the normobaric environment. Recovery kinetics was found to be statistically faster $(p<0.05)$ in the hypoxia than in the normoxia environment.

Conclusions: The ATP supply in an anaerobic way was higher in the normoxic environment than in the normobaric environment, and lactate tolerance increased. Having faster oxygen uptake kinetics in the normoxic environment shows that the volume of oxygen deficit is lower. Faster recovery kinetics in normobaric hypoxia shows the positive effects of altitude on recovery.

Key words: normoxia, normobaric hypoxia, cardiovascular, metabolic, oxygen uptake kinetics.

\section{article details}

Article statistics: Word count: 4,888; Tables: 7; Figures: 6; References: 26

Received: September 2021; Accepted: November 2021; Published: December 2021

Full-text PDF: http://www.balticsportscience.com

Copyright @ Gdansk University of Physical Education and Sport, Poland

Indexation: Celdes, Clarivate Analytics Emerging Sources Citation Index (ESCI), CNKI Scholar (China National Knowledge Infrastructure), CNPIEC, DOAJ, EBSCO - Central \& Eastern European Academic Source, EBSCO - SPORTDiscus, EBSCO Discovery Service, Google Scholar, Index Copernicus, J-Gate, Naviga (Softweco, Primo Central (ExLibris), ProQuest - Family Health, ProQuest - Health \& Medical Complete, ProQuest - Illustrata: Health Sciences, ProQuest Nursing \& Allied Health Source, Summon (Serials Solutions/ProQuest, TDOne (TDNet), Ulrich's Periodicals Directory/ ulrichsweb, WorldCat (OCLC)

Funding: This research received no specific grant from any funding agency in the public, commercial, or not-for-profit sectors.

Conflict of interests: Corresponding author:

Open Access License: Authors have declared that no competing interest exists.

Celil KAÇOĞLU, Eskişehir Technical University, Sport Sciences Faculty, Department of Coaching Education, Gazipaşa district, 2 Eylul Campus, 26555 Tepebaşı Eskişehir/Turkey; phone: 0902223213550 / 6792; e-mail: ckacoglu@ eskisehir.edu.tr

This is an open access article distributed under the terms of the Creative Commons Attribution-Non-Commercial-NoDerivatives 4.0 International (https://creativecommons.org/licenses/by-nc-nd/4.0/), which permits use, distribution, and reproduction in any medium, provided the original work is properly cited, the use is non-commercial and is 


\section{INTRODUCTION}

The physical conditions and game rules are constantly renewing themselves in all competitive sports disciplines, which has led sports scientists to search to improve current performance. Since endurance performance, which is one of the motor skills, is one of the basic criteria that should be gained for many sports, many studies have been done on it, and different methods have been developed. One of the most important of these methods includes training at high altitudes.

In recent years, settlements in high altitude areas and tourist trips to these areas have increased. In addition, there has been an increase in travels to high altitude regions (2000$4000 \mathrm{~m}$ ). Therefore, acute adaptations of travelers to these areas and the chronic adaptations of approximately 150 million people worldwide residing in residential areas $>2500$ meters have attracted researchers' attention [1]. In addition, the effect of physical training at medium altitudes attracts great attention from athletes seeking new methods to improve their sea level performance [2]. Many researchers have tried to find a method that would provide optimum benefit in the relationship between training and altitude [3-6]. Although it is certain that physical activity at altitudes improves performance, research on whether training at high altitudes causes an increase in sea level performance is ambiguous and insufficient [7].

Intermittent exposure to hypoxia has been developed as a new technique in altitude training [8]. Among these developed techniques, the "live at high, train at low" (LHTL) method developed by Levine and colleagues stands out as a combined method that includes training at low altitudes and acclimatization at average altitudes [9]. There is evidence that the LHTL method at natural elevation improves endurance performance [10].

The difficulties that occur in the application of altitude training, the effect of which has been proven by scientific studies, in the natural environment has pushed people to different searches. To overcome this problem, the researchers created "Elevation chambers" in which the oxygen content was reduced using nitrogen. This environment can simulate the necessary altitude conditions for athletes to live, sleep and exercise [4]. Various devices have been used to examine the effect of simulated high altitude on the athlete's performance at sea level. They observed that the change in cycling and running performance at sea level in such programs increased from very small values to $3.7 \%$ when compared to traditional programs [4].

Athletes exposed to hypoxia first experience a decrease in $\mathrm{VO}_{2}$ max values, which can be observed even at altitudes as low as 580 meters [4]. It has been reported that the maximum decreases in $\mathrm{VO}_{2}$ max are 1.5-3.5\% for every 300 meters after 1500 meters, according to various individual characteristics, such as the individual's training or performance level [11]. Athletes' acclimatization to natural elevations $\left(1800-2300 \mathrm{~m}\right.$ ) is a $2-5 \%$ increase in sea level $\mathrm{VO}_{2} \mathrm{max}$, which initially declines to $80-85 \%$ levels, over a $2-3$ week period. Studies with control groups are recommended to determine the developments in $\mathrm{VO}_{2}$ max at sea level. The existence of studies showing that training in altitude conditions has an effect on $\mathrm{VO}_{2}$ max as well as studies showing that it has no effect keep this topic up to date. There is not enough scientific research on the effects of LHTL protocols on $\mathrm{VO}_{2}$ max under natural or artificial elevation conditions [4, 5].

Hahn et al. [5] in their study on elite cycling and ski athletes found that although there was no increase in $\mathrm{VO}_{2}$ max or Hbmass values, the performance showed a statistically insignificant increase trend in tasks lasting an average of 4 minutes. They concluded that sleeping more than 23 days (night sleep) at moderate elevations (2650-3000 m) may have practical benefits for elite athletes, but these beneficial effects do not include the Hbmass and $\mathrm{VO}_{2}$ max values. Jensen et al. [12], in their study on rowing athletes, showed that a 3-week training program at the altitude of 1822 meters did not change the $\mathrm{VO}_{2} \max$ and 6-minute work capacity on a rowing ergometer at sea level; on the contrary, the $\mathrm{VO}_{2}$ max values of the control group training at sea level increased by $4 \%$ and work capacity also increased by $3 \%$. 
Another explanation for the differences between the studies is that there are differences between the initial performances of the athletes. There are hypotheses that athletes with low $\mathrm{VO}_{2}$ max values will improve more in $\mathrm{VO}_{2}$ max change rates that occur as a result of altitude training compared to athletes with high $\mathrm{VO}_{2}$ max values. In addition to the performance level of the athlete before being exposed to the altitude, the amount of altitude and the effect of the protocols applied on the expected performance come to the fore.

In this context, the use of control groups for comparison is important in investigating whether hypoxic conditions have an effect on performance. In some studies where this type of control factor was not included, it was revealed that 3-4 weeks' acclimatization process to an altitude of 1800-2300 meters caused $2-4 \%$ increases in performance; however, no change was observed in the 20-day period [4].

The impact of adaptation to hypoxia on athletes' sea level performance has been a controversial issue for more than 30 years. Although there is no definitive evidence that living or training at altitude (LHTL) improves performance, some sources show that the LHTL approach has positive effects on performance [4]. During Olympic rowing and canoeing activities, athletes stay under a load for 30 seconds to 8 minutes. For this reason, kayakers need maximum strength and muscle power with high maximal aerobic and anaerobic capacity [13]. Hence, a high level of phosphogen production is required at the beginning of the canoe race and the energy metabolism of the anaerobic glycolysis system towards the end [14].

The aim of this study is to evaluate the cardiovascular, metabolic and oxygen uptake kinetic responses to rowing exercises on a kayak ergometer performed in normoxic and normobaric hypoxia (NH). In this context, two general hypotheses have been defined.

I. Cardiovascular and metabolic responses are different in normobaric hypoxia compared to exercise performed in a normoxic environment.

II. In normobaric hypoxia, the oxygen uptake kinetics slows down, and the economy of movement is different compared to the exercise performed in the normoxic environment.

\section{MATERIALS AND METHODS}

\section{PARTICIPANTS}

Seven flatwater kayakers were involved in the study. The physical and physiological characteristics of the subjects with a four-year kayak history are given in Table 1. Subjects participated in the study voluntarily. There is no lactate record because the subject indicated with number four did not want to have his/her blood taken.

Table 1. Demographic Characteristics of kayaker

\begin{tabular}{|c|c|c|c|c|c|c|c|}
\hline $\begin{array}{l}\text { Participant } \\
\text { no }\end{array}$ & Age (year) & Height $(\mathrm{cm})$ & Weight (kg) & $\begin{array}{c}\text { Body Fat } \\
(\%)\end{array}$ & $\begin{array}{l}\mathrm{VO}_{2} \max \\
\left(\mathrm{L}^{2} \mathrm{dk}^{-1}\right)\end{array}$ & $\begin{array}{c}\mathrm{VO}_{2} \max \\
\left(\mathrm{ml} \cdot \mathrm{kg}^{-1} \cdot \mathrm{dk}^{-1}\right)\end{array}$ & $\underset{(\mathrm{mM})}{\operatorname{Maximal} L a}$ \\
\hline 1 & 17 & 180 & 57.1 & 11 & 3.12 & 50 & 11.99 \\
\hline 2 & 17 & 172 & 54 & 13.4 & 1.68 & 27.5 & 8.22 \\
\hline 3 & 15 & 160 & 40 & 23.2 & 1.46 & 29 & 3.97 \\
\hline 4 & 14 & 150 & 50 & 19.1 & 1.08 & 27.4 & - \\
\hline 5 & 16 & 158 & 62 & 21.2 & 1.51 & 30 & 9.2 \\
\hline 6 & 17 & 168 & 54 & 18.6 & 2.03 & 32.7 & 8.29 \\
\hline 7 & 15 & 172 & 61 & 11.5 & 2.45 & 45.4 & 10.66 \\
\hline Mean & 16 & 165 & 53 & 17 & 1.90 & 34.57 & 8.72 \\
\hline \pm S.D. & 1.2 & 8.3 & 8 & 4.4 & 0.69 & 9.24 & 2.75 \\
\hline
\end{tabular}




\section{RESEARCH DESIGN}

All exercise tests were performed at a temperature in the range of $21-22^{\circ} \mathrm{C}$. All subjects had previously trained on a kayak ergometer. Preliminary trials related to all tests were applied in the first week. In the second week, as indicated in Figure 1, physiological responses were evaluated in four separate tests: the augmented test in the normoxic condition, the anaerobic sub-threshold test with a constant load, and the same test in hypoxia.

\begin{tabular}{|l} 
1. Week \\
Preliminary trials of test protocols
\end{tabular} \mid $\begin{aligned} & \text { Week } \\
& \text { 1-Augmented maximal test under } \\
& \text { normoxic condition } \\
& \text { 2-Anaerobic sub-threshold test } \\
& \text { with constant load under } \\
& \text { normoxic condition } \\
& \text { 3- Augmented maximal test in } \\
& \text { normobaric hypoxia condition }\end{aligned}$

Fig. 1. Flowchart of the study

Kayakers performed the maximal incremental tests on a kayak ergometer (Dansprint, UK) (Figure 2). The test protocol started with rowing at $25 \mathrm{~W}$ workload for 2 minutes and continued with $25 \mathrm{~W}$ increments every two minutes, and ended at the individual exhaustion point [15]. The specified test protocol was applied in both normoxic and normobaric hypoxia environments [15]. All subjects were motivated to show their maximal performance. It has been determined as the point at which there was a break in the linearity of the $\mathrm{VCO}_{2}$ compared to $\mathrm{VO}_{2}$ curve. The anaerobic threshold (respiratory threshold) was determined by the $\mathrm{V}$ slope method. It was found that the linearity of the $\mathrm{VCO}_{2}$ and $\mathrm{VO}_{2}$ curve is disrupted and the break is the place [16]. Individual $\mathrm{VO}_{2} \max$ was calculated in line with the criteria for achieving $\mathrm{VO}_{2}$ max stated in the literature. Performance tests were carried out in an airconditioned laboratory environment and at the same time of day ( 2 hours) $\left(18^{\circ} \mathrm{C}\right)$. Blood lactate ([La]) samples were taken at rest and at the end of the test [17]. Oxygen consumption was measured with the Masterscreen CPX (Germany) device (Figure 2). Gas calibration had been done. During the test, the heart rates of the athletes were recorded with the device (Model, Polar, Finland).
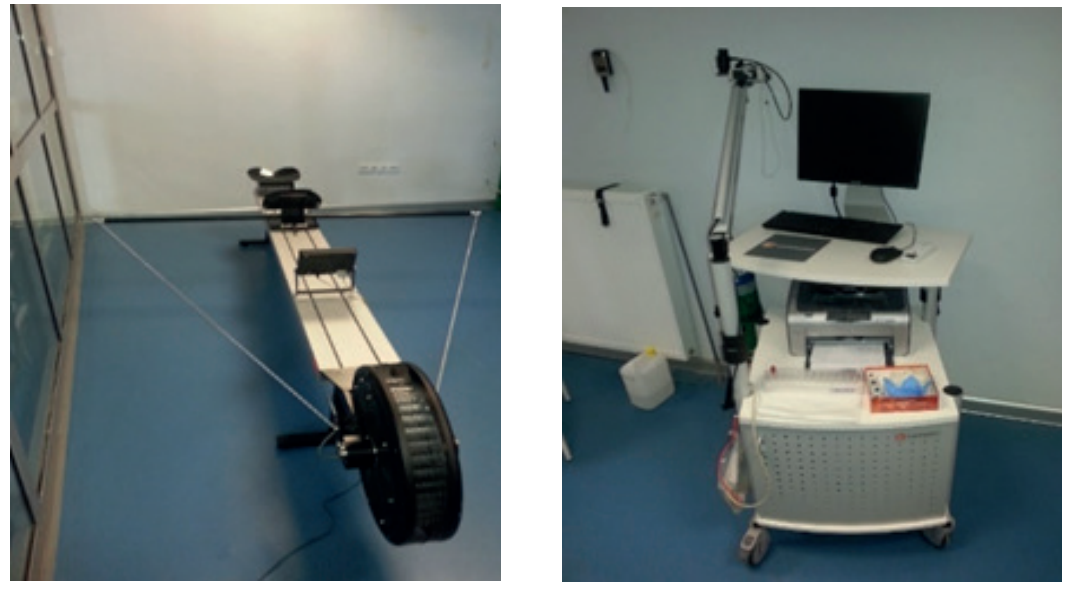

Fig. 2. Kayak ergometer and oxygen analyzer 


\section{MODELLING OF OXYGEN UPTAKE KINETICS}

A constant load anaerobic sub-threshold test was applied at $90 \%$ AE exercise intensity. After taking 3 minutes of rest data, oxygen consumption was recorded during rowing on a kayak ergometer at a workload (W) corresponding to the $90 \% \mathrm{AE}$ point for 6 minutes. After the completion of the performance, 5 minutes of recovery data were taken.

The $\mathrm{VO}_{2}$ data consumed during individual rest was calculated by averaging the data between 2-3 minutes of the first three minutes. Because the performance is below AE, there is a balance between the accumulation of lactate and its removal from the blood. Therefore, in accordance with the literature, oxygen consumption increases to meet the increasing need for ATP-CP with the start of exercise and reaches equilibrium in about three minutes [18].

There is a square wave increase in workload, while there is a delay in the response of oxygen consumption. The speed of reaching steady-state and the steady-state amplitude of the $\mathrm{VO}_{2}$ graph vary both individually and according to the altitude measured. In order to calculate these parameters, the $\mathrm{VO}_{2}$ graph was modeled by adding intermediate values (iteration), curve fitting, and a single exponential function. The order of the applied procedures is listed below:

First of all, a scatter diagram of $\mathrm{VO}_{2}$ responses to incremental maximal exercise was drawn as seen in Figure 3.

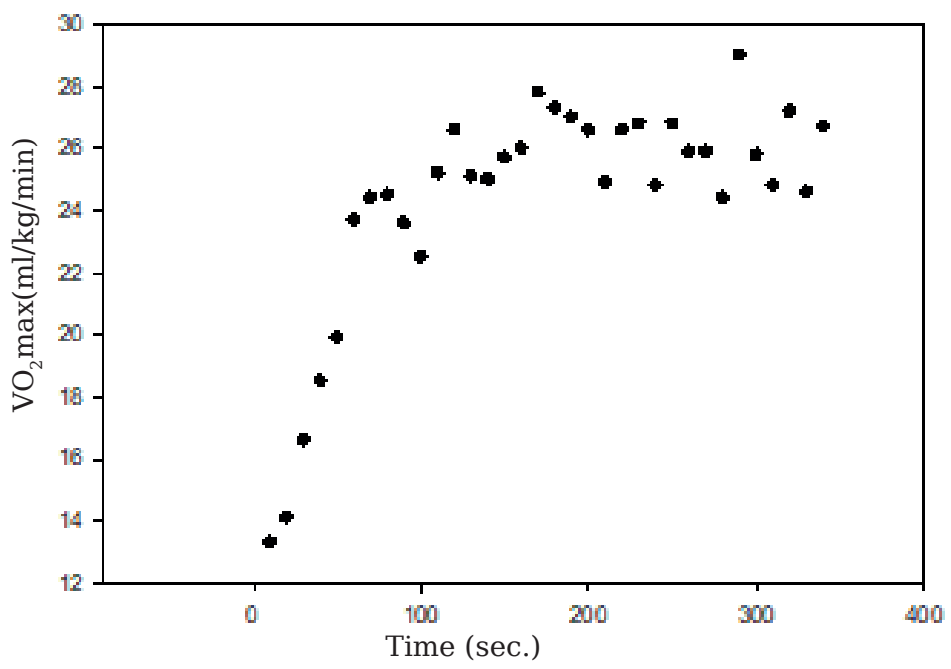

Fig. 3. Scattering diagram of $\mathrm{VO}_{2}$ response to incremental maximal exercise vs. time

Oxygen consumption data were interpolated by adding intermediate values, and curve fitting was performed. In the modeling, a two-parameter increasing single exponential equation was used. The actual data (scatter diagram) and the modeled curve are shown in Figure 4. As seen in Table 2, R, $\mathrm{R}^{2}$, a and b parameters were taken into account in the final report of the nonlinear regression analysis. It was evaluated if the $\mathrm{R}$ value showing the ratio of the modeled data reflecting the real data was above $85 \%$ and the a and $\mathrm{b}$ parameters were statistically significant. 


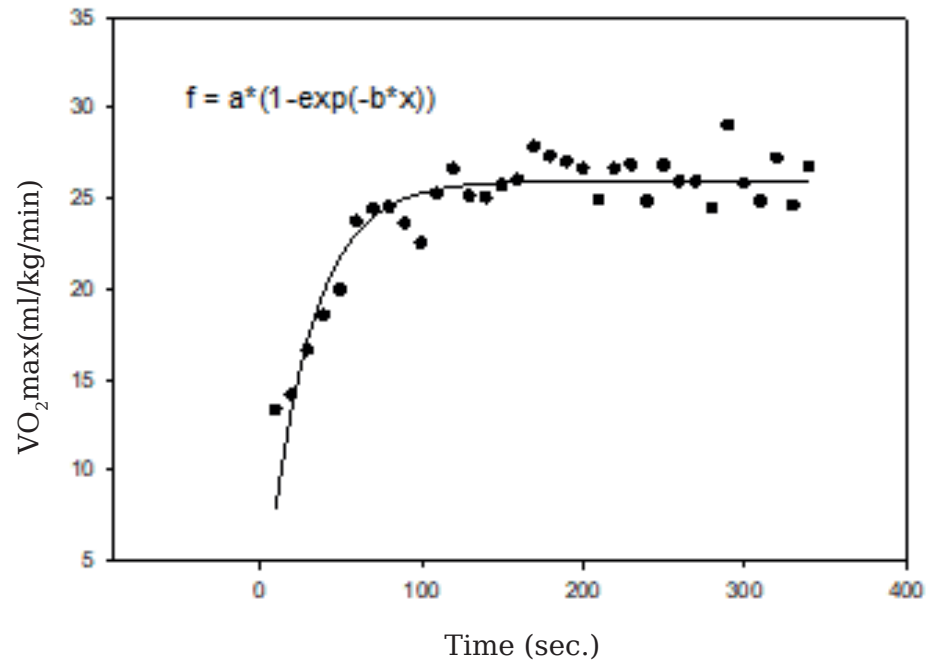

Fig. 4. Modeling of the $\mathrm{VO}_{2}$ response to augmented exercise with curve fitting with increasing exponential function

Table 2. Parameters examined in the results table of best curve fitting with nonlinear regression

\begin{tabular}{cc}
$\mathrm{R}$ & $\mathrm{R}^{2}$ \\
\hline 0.91 & 0.85 \\
$\mathrm{~A}$ & $\mathrm{P}<0.05$ \\
$\mathrm{~B}$ & $*$ \\
\hline $\mathrm{VO}_{2}(\mathrm{t})=\mathrm{A} 1 \times\left(1-\mathrm{e}^{-\mathrm{kt}}\right)$
\end{tabular}

The 'A1' parameter (shown as a in Table 2) for the steady state amplitude of the graph, and the inverse of the rate constant ' $\mathrm{k}$ ' (shown as $\mathrm{b}$ in Table 2) in the function to calculate the time constant giving the steady state speed of the graph [18].

$$
1 / \mathrm{k}=\text { time constant }(\mathrm{tau})
$$

According to the individual AE point in the incremental maximal test result, the $90 \% \mathrm{AE}$ workload was determined individually. Therefore, one subject performed the fixed load test at $25 \mathrm{~W}$ workload, while another performed at $55 \mathrm{~W}$ workload. Since it would be more accurate to make a proportional evaluation instead of A1 in order to compare the performance at different heights, the $\mathrm{O}_{2}$ value was evaluated by calculating the ratio of the individual A1 value to the workload (W).

\section{ANALYSIS OF OXYGEN UPTAKE KINETIC RESPONSES IN RECOVERY}

After the completion of the six-minute exercise, $\mathrm{VO}_{2}$ was recorded for five minutes. $\mathrm{VO}_{2}$, which increases with exercise, decreases with the termination of exercise and returns to resting values. Oxygen uptake kinetics were modeled to determine the speed and amplitude of this reduction.

First of all, the scatter plot of the decreasing graph of the $\mathrm{VO}_{2}$ data in the 5-minute period after the end of the exercise was drawn (Figure 5). 


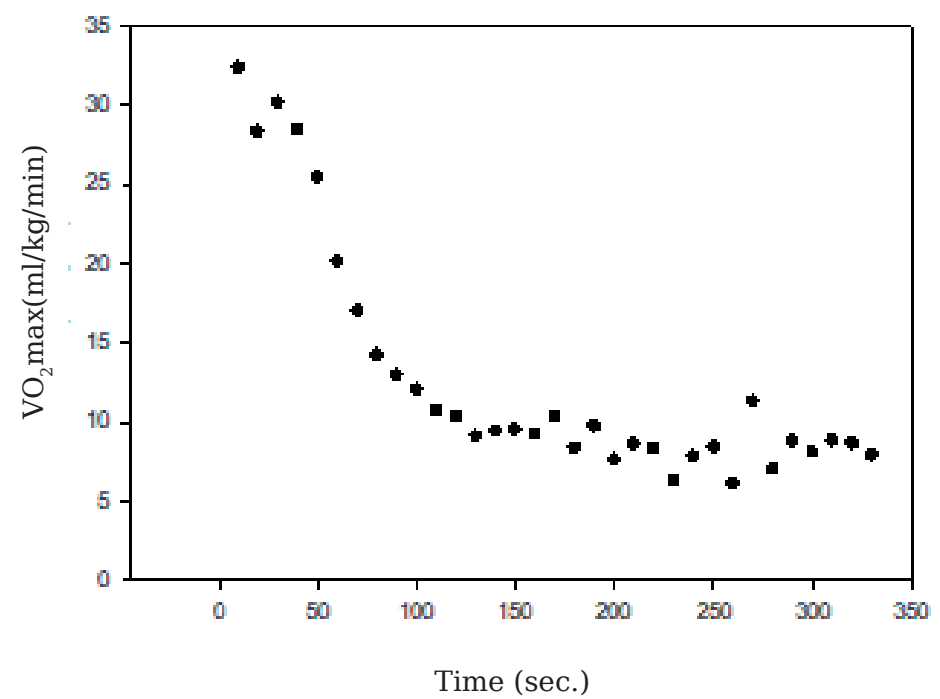

Fig. 5. Scattering diagram of oxygen consumption during recovery vs. time

$\mathrm{VO}_{2}$ data is modeled with a 3-parameter decreasing single exponential function. The function used is given below. While the measured $\mathrm{VO}_{2}$ data consisted of 33 points, 200 points were added to the intervals with the iteration process applied (inserting intermediate values). As seen in Table 3, $\mathrm{VO}_{2}$ data taken at 10 -second intervals is modeled with interval time values such as $11.25,12.50,13.75,15,16.25,17.50$ and $\mathrm{VO}_{2}$ data that may correspond to these values. These calculations are given by the sigma plot program where the analyses are made.

Table 3. Explanation of curve fitting operation with interpolation (adding intermediate values) operation

\begin{tabular}{cccc} 
& \multicolumn{2}{c}{ Real Data } & \multicolumn{2}{c}{ Modified Data } \\
Time $(\mathrm{s})$ & $\mathrm{VO}_{2}\left(\mathrm{ml}_{\mathrm{kg}} \mathrm{kg}^{-1} \mathrm{dk}^{-1}\right)$ & Time $(\mathrm{s})$ & $\mathrm{VO}_{2}\left(\mathrm{ml}^{\mathrm{k}} \mathrm{kg}^{-1} \cdot \mathrm{dk}^{-1}\right)$ \\
\hline 10.000 & 32.300 & 10.000 & 35.107 \\
20.000 & 28.300 & 11.250 & 34.523 \\
30.000 & 30.100 & 12.500 & 33.952 \\
40.000 & 28.400 & 13.750 & 33.393 \\
50.000 & 25.400 & 15.000 & 32.846 \\
60.000 & 20.100 & 16.250 & 32.311 \\
70.000 & 17.000 & 17.500 & 31.786 \\
80.000 & 14.200 & 18.750 & 31.273 \\
\hline
\end{tabular}

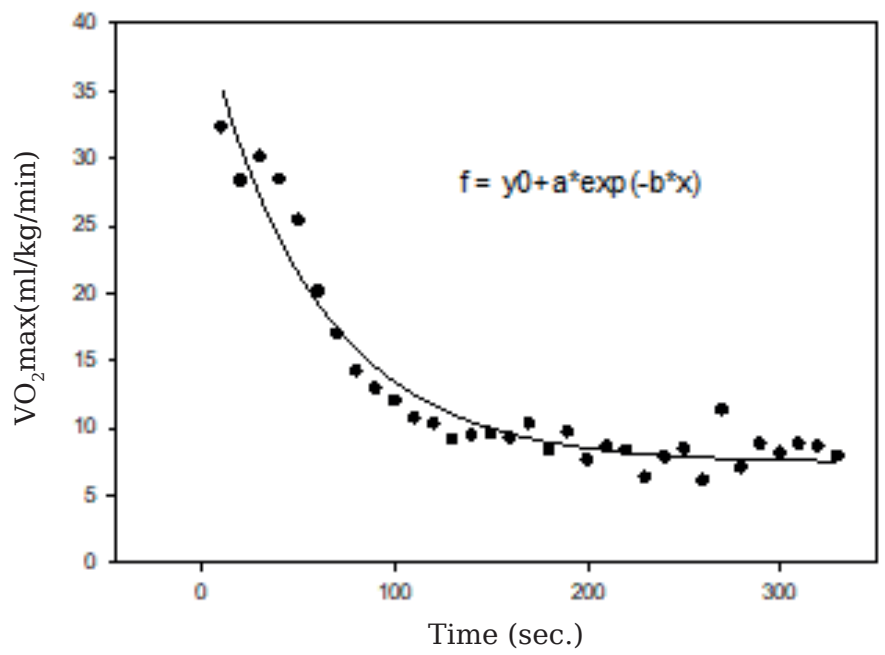

Fig. 6. Modeling of oxygen consumption during recovery by curve fitting with decreased exponential function 
The curve modeled by the actual $\mathrm{VO}_{2}$ (scatter diagram) that the person consumes after the completion of the exercise and the increasing 2-parameter single exponential function is shown in Figure 6. The function used in the modeling is given below.

$$
f=y 0+\left(a \times e^{-b x}\right)
$$

Table 4. Parameters examined in the result table of curve fitting with nonlinear regression

\begin{tabular}{cc}
$\mathrm{R}$ & $\mathrm{R}^{2}$ \\
\hline 0.97 & 0.94 \\
& $\mathrm{P}<0.05$ \\
$\mathrm{y} 0$ & $*$ \\
$\mathrm{a}$ & $*$ \\
\hline
\end{tabular}

Looking at the result table of the modeled data, it was checked whether the $\mathrm{R}$ value was greater than 0.85 as seen in Table 4 . An $\mathrm{R}$ value greater than $85 \%$ indicates that the modeled data reflects the real data at the rate of $85 \%$, and this ratio is accepted. However, it is expected that the analyzed parameters will show statistical significance. As a result, if the modeled data reflects the real data at a rate of $85 \%$ and/or more, and the investigated parameters (y0, a, b) are statistically significant, the parameters were recorded. All analyses were calculated separately for each individual and the results obtained are given in Tables 5 and 6 both individually and as an average.

\section{RESULTS}

Cardiovascular and metabolic responses to the maximal boost kayak test in normoxic and normobaric hypoxia environments are given in Table 5.

Table 5. Comparison of cardiovascular and metabolic responses in normoxic and NH environment

\begin{tabular}{|c|c|c|c|c|}
\hline Parameters & $\begin{array}{l}\text { Normoxic Test } \\
\quad(M \pm S D)\end{array}$ & $\begin{array}{c}\text { Normobaric Hypoxia Test } \\
(M \pm S D)\end{array}$ & $\%$ & $\mathrm{P}$ \\
\hline $\mathrm{VO}_{2} \max \left(\mathrm{L} \mathrm{dk}^{-1}\right)$ & $1.90 \pm 0.7$ & $1.85 \pm 0.7$ & 3 & * \\
\hline $\mathrm{VO}_{2} \max \left(\mathrm{ml} \cdot \mathrm{dk}^{-1} \cdot \mathrm{kg}^{-1}\right)$ & $34.2 \pm 9.5$ & $34.6 \pm 9.2$ & 1 & * \\
\hline $\mathrm{V}_{\mathrm{E}} \max \left(\mathrm{L} \cdot \mathrm{dk}^{-1}\right)$ & $71 \pm 25.4$ & $77.3 \pm 19.5$ & 9 & * \\
\hline Max. HR (bpm) & $188 \pm 13$ & $184 \pm 12$ & 2 & $*$ \\
\hline Pike power (W) & $72 \pm 25$ & $70 \pm 21$ & 3 & $*$ \\
\hline Max. Lactate (mmol.L-1) & $8.72 \pm 2.75$ & $7.48 \pm 1.95$ & 14 & $*$ \\
\hline
\end{tabular}

(Mean $\pm \mathrm{SE}) ; \mathrm{VO}_{2}$ max: highest oxygen consumption evaluated as average in the last 30 seconds of the test; Max HR: heart rate evaluated as average over the last 30 seconds of the test; $V_{E} \max$ : ventilation evaluated as average over the last 30 seconds of the test; Max Lactate: lactate at the end of the test

When $\mathrm{VO}_{2}$ max was evaluated both in absolute (L.min ${ }^{-1}$ ) and proportionally $\left(\mathrm{ml} \cdot \mathrm{min}^{-1} \cdot \mathrm{kg}^{-1}\right)$ in the test performed under normoxic conditions, it was found to be higher than the test performed in normobaric hypoxia $(\mathrm{P}<0.05)$. Similarly, VEmax was found to be statistically higher in the normoxic test than in the normobaric test $(\mathrm{P}<0.05)$, at peak power, which was evaluated as the maximal workload at the end of the test. The maximal heart rate at the end of the incremental test was statistically higher at the end of the normoxic test than at the normobaric hypoxia test $(\mathrm{P}<0.05)$. The maximal lactate value, which is the parameter in which the metabolic response to the test is evaluated, was found to be higher in the normoxic test than in the normobaric hypoxia test $(\mathrm{P}<0.05)$. 
Table 6. Oxygen uptake kinetic responses to constant-load anaerobic subthreshold exercise in normoxic and normobaric hypoxic conditions

\begin{tabular}{|c|c|c|c|c|c|c|c|c|c|c|}
\hline \multirow[t]{2}{*}{$\begin{array}{c}\text { Participant } \\
\text { no }\end{array}$} & \multicolumn{2}{|c|}{$\begin{array}{l}\text { Work load } \\
\text { (W) }\end{array}$} & \multicolumn{2}{|c|}{$\begin{array}{c}\text { Rest VO } \\
\left(\mathrm{ml}^{2} \mathrm{~kg}^{-1} \mathrm{dk}^{-1}\right)\end{array}$} & \multicolumn{2}{|c|}{$\begin{array}{c}\text { Time constant } \\
\text { (s) }\end{array}$} & \multicolumn{2}{|c|}{$\begin{array}{l}\text { Amplitude } \\
\left(\mathrm{L}^{\left.\mathrm{d} \mathrm{dk}^{-1}\right)}\right.\end{array}$} & \multicolumn{2}{|c|}{$\begin{array}{c}\mathrm{O}_{2} \text { value } \\
\left(\mathrm{ml} \mathrm{dk}^{-1} \mathrm{~W}^{-1}\right)\end{array}$} \\
\hline & NT & NHT & NT & NHT * & NT & NHT * & $\mathrm{NT}$ & NHT * & NT & NHT * \\
\hline 1 & 40 & 40 & 6.8 & 7.9 & 27 & 29 & 1.47 & 1.47 & 26.7 & 25.5 \\
\hline 2 & 40 & 40 & 4.5 & 6.5 & 27 & 39 & 1.43 & 1.62 & 29 & 31 \\
\hline 4 & 25 & 25 & 6.7 & 7.6 & 25 & 27 & 1.05 & 1.18 & 31.6 & 36.4 \\
\hline 5 & 25 & 25 & 6 & 6.3 & 25 & 29 & 1.32 & 1.44 & 39.9 & 43.9 \\
\hline 6 & 55 & 55 & 8.8 & 10.4 & 23 & 32 & 1.95 & 1.99 & 27.9 & 27 \\
\hline$M$ & 35 & 35 & 6.6 & 7.9 & 25 & 30 & 1.42 & 1.53 & 32.4 & 34.3 \\
\hline
\end{tabular}

(Mean $\pm S E)$; NT: normoxic test $(n=4)$; NHT: normobaric hypoxic test

Oxygen consumption graphs of subjects performing at $90 \%$ of the individual AE values in the incremental test result were modeled by performing curve-fitting analysis with a single exponential function (Sigma Plot). The function used to determine the oxygen uptake kinetic parameters is given below.

$$
\mathrm{VO}_{2}(\mathrm{t})=\mathrm{A} 1 *\left(1-\mathrm{e}^{-\mathrm{kt}}\right)
$$

In the final report of the modeled individual $\mathrm{VO}_{2}$ data, the $\mathrm{R}$ value and the significance of the parameters $(\mathrm{A} 1, \mathrm{k})(\mathrm{p}<0.05)$ were examined. It presents the $\mathrm{R}$ value, which shows the ratio of the modeled data to the real data. If the $\mathrm{R}$ value was above 0.85 , and the significance level of the parameters was $\mathrm{p}<0.05$, the parameters were evaluated. The parameter 'A1' specified in the function gives the amplitude at which the oxygen consumption of individuals reaches the steady state. The 'A1' parameter is given as 'amplitude' in the table. The ' $k$ ' parameter is used to calculate the rate of oxygen uptake kinetics in the function. The parameter ' $k$ ' in the function gives the rate constant. When the inverse of the rate constant is taken, the time constant is calculated. Both the individual and average values of the oxygen uptake kinetic responses in the sub-threshold (90\% $\mathrm{AE}$ ) test with a constant load are given in Table 6. Subject numbers are given in the first column of Table 6. Individual anaerobic subthreshold workloads did not differ in NT and NHT. However, the $\mathrm{VO}_{2}$ data obtained in the resting state of the individuals before starting the exercise were found to be higher during NHT than during NT. Similarly, time constant, amplitude and $\mathrm{O}_{2}$ value were also higher in NHT.

Table 7. Recovery oxygen uptake kinetic responses to constant-load anaerobic subthreshold exercise under normoxic and normobaric hypoxic conditions

\begin{tabular}{|c|c|c|c|c|c|c|c|c|}
\hline \multirow[t]{2}{*}{$\begin{array}{c}\text { Participant } \\
\text { no }\end{array}$} & \multicolumn{2}{|c|}{$\begin{array}{l}\text { Work load } \\
\text { (W) }\end{array}$} & \multicolumn{2}{|c|}{$\begin{array}{c}\mathrm{y} 0 \mathrm{VO}^{2} \\
\left(\mathrm{ml}^{l} \cdot \mathrm{kg}^{-1} \mathrm{dk}^{-1}\right)\end{array}$} & \multicolumn{2}{|c|}{$\begin{array}{c}\text { Time constant } \\
\text { (s) }\end{array}$} & \multicolumn{2}{|c|}{$\begin{array}{c}\text { Amplitude } \\
\left(\mathrm{L}^{\left.-\mathrm{dk}^{-1}\right)}\right.\end{array}$} \\
\hline & NT & $\mathrm{NHT}$ & NT & NHT * & NT & NHT * & NT & $\mathrm{NHT} *$ \\
\hline 1 & 40 & 40 & 5.5 & 5.3 & 53 & 51 & 20.2 & 20.5 \\
\hline 2 & 40 & 40 & 5.3 & 7 & 58 & 47 & 17.2 & 25 \\
\hline 4 & 25 & 25 & 6.7 & 8 & 47 & 50 & 26.1 & 27.1 \\
\hline 5 & 25 & 25 & 6.2 & 7.8 & 49 & 47 & 22.1 & 27.9 \\
\hline 6 & 55 & 55 & 7.7 & 7.4 & 53 & 59 & 31.9 & 32.8 \\
\hline$M$ & 35 & 35 & 6.3 & 7.3 & 53 & 49 & 23.5 & 25.4 \\
\hline
\end{tabular}

(Mean \pm SE); NT: normoxic test $(n=4)$; NHT: normobaric hypoxic test 
The recovery $\mathrm{VO}_{2}$ data obtained after the completion of the exercise was modeled by performing curve-fitting analysis with a decreasing three-parameter single exponential function (Sigma Plot). The function used to determine the oxygen uptake kinetic parameters is given below.

$$
\mathrm{f}=\mathrm{y} 0+\left(\mathrm{a} \mathrm{x} \mathrm{e}^{-\mathrm{bx}}\right)
$$

The $R$ value and the significance of the parameters $(y 0, a, b)(p<0.05)$ were examined in the final report of the modeled individual $\mathrm{VO}_{2}$ data. It presents the $\mathrm{R}$ value, which shows the ratio of the modeled data to the real data. If the $R$ value was above 0.85 and the significance level of the parameters was $\mathrm{p}<0.05$, the parameters were evaluated. The 'A1' parameter specified in the function gives the amplitude of the oxygen consumption of individuals at the point where the exercise is completed. The 'A1' parameter is given as 'amplitude' in the table. The ' $b$ ' parameter is used to calculate the rate of oxygen uptake kinetics in the function. The ' $b$ ' parameter in the function gives the rate constant. When the inverse of the rate constant is taken, the time constant is calculated. Both the individual and average values of the oxygen uptake kinetic recovery responses in the sub-threshold (90\% AE) test with constant load are given in Table 7 . Subject numbers are given in the first column of Table 7. Individual anaerobic subthreshold workloads did not differ in NT and NHT. However, the oxygen consumption values (y0) at the time of completion of the exercise were found to be higher during NHT compared to NT. Similarly, time constant and amplitude values were found higher in NHT.

\section{DISCUSSION}

In order to perform altitude training, which is used for endurance development, appropriate facilities, living standards and materials are often required. As environments with these standards cannot be easily provided, and with the development of technology, artificial environments where hypoxic conditions are provided have been established [19]. As a result of studies conducted on rats, it has been stated in the literature that "the live at height and train at height (LHTH)" approach does not increase performance at sea level, and that the LHTH approach contributes to performance [20]. In a study conducted on humans, it was stated that the 4-week SCA approach had no effect on endurance performance or physiological parameters [21].

Within the scope of this research, it was found that the physiological responses in an altitude chamber $(3000 \mathrm{~m})$, where altitude can be artificially provided during canoe exercise, differ from the physiological responses in tests performed under normoxic conditions. Physiological responses during hypoxic exposure may be due not only to partial oxygen pressure in inspired gas $\left(\mathrm{P}_{1} \mathrm{O}_{2}\right)$, but also to the methodological difference in establishing hypoxic conditions, i.e. a reduction in atmospheric $\mathrm{O}_{2}$ concentration or barometric pressure [22].

When exercising at altitude, the body has to respond to two separate stresses: exercise and hypoxia. The factors that affect the size of the body's response to exercise and hypoxia stresses are stated as the person's performance level, height and individual characteristics [23]. Since the subjects participating in this study were athletes of the same team, they did not differ in terms of training levels and ages. Therefore, in this study, the effect of altitude on physiological responses could be determined.

One of the most important factors that reveal the aerobic performance of athletes is the capacity to transmit oxygen to the working tissues. It has been demonstrated for many years that endurance training increases blood volume in both men and women [24]. Subsequent studies concluded that this development was due to greater erythrocyte and plasma volume. 
Although blood volume adaptation refers to only a few mechanisms that allow for maximal aerobic performance improvement, it has been proven to be related to maximal oxygen uptake $\left(\mathrm{VO}_{2} \max \right)[25]$.

Most of the studies conducted in the altitude environment have observed the changes in the blood values, heart rates and maximal oxygen consumption capacities of the athletes. However, within the scope of this research, oxygen uptake kinetics of individuals were investigated depending on loading at high and low altitudes.

The main finding of this research is that $\mathrm{VO}_{2} \max$, maximal $\mathrm{HR}$, peak power $(\mathrm{W})$ and maximal lactate values differed in tests performed in normobaric hypoxia and normoxic environment. The $\mathrm{VO}_{2} \max \left({\left.\mathrm{L} . \mathrm{min}^{-1}\right)}^{-}\right.$response was found to be higher in the test performed in the normoxic environment compared to the measurement performed in normobaric hypoxia. Since there is a physiological strain as the altitude increases, it is expected that the $\mathrm{VO}_{2}$ max $\left(\mathrm{L} \cdot \mathrm{min}^{-1}\right)$ response will decrease, while the fact that the $\mathrm{VO}_{2} \max \left(\mathrm{ml} . \mathrm{kg}^{-1} \cdot \mathrm{min}^{-1}\right)$ response, which is evaluated proportionally, is higher in the normobaric hypoxia environment does not support this finding. Therefore, a clear judgement could not be reached based on the findings obtained as a result of the evaluation of the $\mathrm{VO}_{2}$ response in the normoxic and normobaric hypoxia environment in this study. Maximal heart rate responses were found to be higher in the normoxic test than in the normobaric test. Subjects who took the test in normoxic conditions were able to perform at higher heart rates. If the researched group were more trained and older in age, no difference would be expected, since they would already be individuals who could reach the maximal heart rate. However, a difference in the heart rate is an expected situation since the subjects cannot reach their maximal heart rate anyway due to the small age group and low training level of the subjects. The peak power value was found to be higher than the normobaric hypoxia environment because the exercise performed in normoxic conditions was less challenging. The fact that the maximal lactate values are higher in the normoxic test can be explained as showing that lactate tolerance is higher in the normoxic test. Individual fixed load AEAE (90\% AE) workloads do not differ as a result of the incremental test applied in normoxic and normobaric hypoxia environments. Although the cardiovascular and metabolic responses in exercises performed in normoxic and normobaric hypoxia environments statistically differed, this difference was not reflected in the performed workload. Oxygen consumption values at rest as assessed by the constant load test $\mathrm{NH}$ was found to be higher in normobaric hypoxia than in normoxic environment. It is thought that the decrease in oxygen in the environment at altitude may force people to consume more oxygen. Oxygen uptake kinetics are $\mathrm{NH}$ in normobaric medium. Oxygen uptake kinetics (time constant) are faster in normoxia than $\mathrm{NH}$.

In another study including 8 people with exercise intensity above $\mathrm{AE}$

$$
\left[\Delta 50 \%=\left(\mathrm{AE}+50 \%\left(\mathrm{VO}_{2} \max -\mathrm{VO}_{2}(\mathrm{AE})\right)\right)\right],
$$

it was concluded that $\mathrm{VO}_{2}$ kinetics slowed down in the hypoxic environment. Although the exercise intensity is not the same, the results are parallel [26]. Faster oxygen uptake kinetics means less oxygen deficit, less ATP-CP used at the beginning of exercise, and energy can be supplied from the aerobic system more quickly [18]. It is stated in the literature that the energy consumption increases at a certain rate (time constant) in fixed load AEAEs [18]. The amplitude of the steady state oxygen consumption and the $\mathrm{O}_{2}$ value obtained by proportioning this amplitude to the applied workload shows the economy of motion. According to the results, it can be said that the effects of $\mathrm{NH}$ environment results the slowing oxygen dynamics and declining exercise capacity. 
As a result of this research, in the exercise performed under normobaric conditions, $\mathrm{NH}$ it is seen that better performance can be achieved compared to the exercise performed in the environment. As a result of more ambient oxygen, NH more oxygen consumption, higher heart rates, higher lactate values and more time performance were realized according to the conditions. Along with these findings, oxygen uptake kinetic responses evaluated with a sudden load given to the organism were also determined by $\mathrm{NH}$ it is considered to be faster than the environment. This means that people were able to adapt to the sudden load more quickly, and their physiological responses were faster. Movement economics is also $\mathrm{NH}$ found better than the environment. This finding shows that in normobaric environment, the organism can do this job using less $\mathrm{O}_{2}$ when faced with a constant loaded stress. As a result, in the exercise performed in normoxic environment, exercise economy or efficiency refers to the VO2 amplitude in constant load exercise is higher than $\mathrm{NH}$

As a result, performance of kayak exercise test in $\mathrm{NH}$ is more challenging compared to the $\mathrm{VO}_{2}$ responses under normoxic conditions. The fact that the exercise to be applied under these conditions causes more stress in the organism compared to the normoxic conditions suggests that the adaptation response may also be faster.

\section{CONCLUSIONS}

In line with the experimental results obtained, it was determined that the exercise performed in $\mathrm{NH}$ environment had more physiological strain than normoxia environment. It is known that exercise causes a stress in the human organism. Therefore, with exercise, $\mathrm{NH}$ environment increases this stress, and the resulting physiological strain may cause higher responses in terms of adaptation. As a result, $\mathrm{NH}$ can be recommended for athletes improving performance. However, these research findings only include physiological responses that occur during exercise at $3000 \mathrm{~m}$ altitude. The following topics can be suggested for future research.

1. Training in harsh conditions can result in a higher level of adaptation. Exercising at 3000 $\mathrm{m}$ in a simulated elevation room can improve performance at sea level. So physiological adaptations to 6 weeks of exercise at NH can be investigated via longitudinal studies.

2. Physiological responses at different heights can be compared.

3. Physiological responses during cycling and running exercises at different heights can be evaluated.

4. The effect of aerobic exercises in the NH environment on cardiovascular and metabolic parameters, oxygen uptake kinetics and performance criteria specific to the applied sports discipline can be evaluated.

5. NH physiological responses in the environment can be evaluated.

\section{REFERENCES}

[1] Schoene RB. Illnesses at High Altitude. Chest. 2008;134:402-416. https://doi.org/10.1378/chest.07-0561

[2] Clark SA, Aughey RJ, Gore CJ, et al. Effects of live high, train low hypoxic exposure on lactate metabolism in trained humans. J Appl Physiol. 2004;96(2):517-525. https://doi.org/10.1152/japplphysiol.00799.2003

[3] Levine BD, Stray-Gundersen J. Living High-Training Low: Effect of Moderate-Altitude Acclimatization with Low-Altitude Training on Performance. J Appl Physiol. 1997;83:102-112. https://doi.org/10.1152/jappl.2001.91.3.1113

[4] Hahn AG, Gore CJ. The Effect of Altitude on Cycling Performance: A Challenge to Traditional Concepts. Sports Med. 2001;31:533-557. https://doi.org/10.2165/00007256-200131070-00008

[5] Henderson KK, Clancy RL, Gonzalez NC. Living and Training in Moderate Hypoxia Does Not Improve VO2 max More Than Living and Training in Normoxia. J Appl Physiol. 2001;90(6):2057-2062. https://doi.org/10.1152/jappl.2001.90.6.2057

[6] Rusko H, Tikkanen H, Peltonen J. Altitude and Endurance Training. J Sports Sci. 2004;22(10):928-945. https://doi. org/10.1080/02640410400005933

[7] Berglund B. High-altitude training. Aspects of haematological adaptation. Sports Med. 1992;14(5):289-303. https://doi. org/10.2165/00007256-199214050-00002

[8] Brugniaux JV, Schmitt L, Robach P, et al. Living high-training low: tolerance and acclimatization in elite endurance athletes. Eur J Appl Physiol. 2006;96(1):66-77. https://doi.org/10.1007/s00421-005-0065-9 
[9] Tiollier E, Schmitt L, Burnat P, et al. Living high-training low altitude training: effects on mucosal immunity. Eur J Appl Physiol. 2005;94:298-304. https://doi.org/10.1007/s00421-005-1317-4

[10] Hinckson EA. Hopkins WG. Changes in running endurance performance following intermittent altitude exposure simulated with tents. Eur J Sport Sci. 2005;5(1):15-24. https://doi.org/10.1080/17461390500077301

[11] Burtscher M. The athlete at high altitude: Performance diminution and high-altitude illnesses. IntJ Sports Med. 2005;6(4):215223. https://doi.org/10520/EJC48579

[12] Jensen K, Nielsen TS, Fiskestrand A, Lund JO, Christensen NJ, Sechef NH. High-altitude training does not increase maximal oxygen uptake or work capacity at sea level in rowers. Scand J Med Sci Sports. 1993;3(4):256-262. https://doi. org/10.1111/j.1600-0838.1993.tb00391.x

[13] Garcia-Pallares J, Izquierdo M. Strategies to Optimize Concurrent Training of Strength and Aerobic Fitness for Rowing and Canoeing. Sports Med. 2011;41(4):329-343. https://doi.org/10.2165/11539690-000000000-00000

[14] Zhu RJ. Body shape analysis of China's canoeing athletes. J Chem Pharm Res. 2014;6(6):1929-1931.

[15] Kerr RM, Spinks W, Leicht AS, Sinclair W, Woodside L. Comparison of physiological responses to graded exercise test performance in outrigger canoeing. J Sports Sci. 2008;26(7):743-749. https://doi.org/10.1080/02640410701791306

[16] Beaver WL, Wasserman KW, Hipp WJ. A new method for detecting anaerobic threshold by gas exchange. J Appl Physiol. 1986;60:2020-2027. https://doi.org/10.1152/jappl.1986.60.6.2020

[17] Dufour SP, Ponsot E, Zoll J, et al. Exercise training in normobaric hypoxia in endurance runners. I. Improvement in aerobic performance capacity. J Appl Physiol. 2006;100:1238-1248. https://doi.org/10.1152/japplphysiol.00742.2005

[18] Jones AM, Poole DC. Introduction to oxygen uptake kinetics and historical development of the discipline, Oxygen uptake kinetics in sport, exercise and medicine. New York: Routledge; 2005, 47-66.

[19] Cerrah AO. Farklı Aerobik Dayanıklılığa Sahip Sporcuların Yükselti Antrenmanına Fiziksel Cevapları ve Optimum Yükseklik ve Kalış Süresi [Physiologic Responses of Different Aerobic Level Athletes to Altitude Training and Optimum Altitude and Exposing Time] Pamukkale J Sport Sci. 2010;1(3):24-38.

[20] Çolak R, Erken HA, Genç O. Sıçanlarda Normobarik Hipoksi Antrenmanlarının Elektrik Uyarısı ile Oluşturulan İskelet Kas Yorgunluğuna Etkisi [The Effect of Normobaric Hypoxic Training on Electrical Stimulation Induced Skeletal Muscle Fatigue in Rats], Pamukkale J Sport Sci. 2013;4:77-84.

[21] Siebenmann C, Robach P, Jacobs RA, et al. Live high-train low using normobaric hypoxia: a double-blinded, placebo-controlled study. J Appl Physiol. 2012;112(1):106-117. https://doi.org/10.1152/japplphysiol.00388.2011

[22] Takezawa T, Dobashi S, Koyama, K. Cardiorespiratory Response and Power Output During Submaximal Exercise in Normobaric Versus Hypobaric Hypoxia: A Pilot Study Using a Specific Chamber that Controls Environmental Factors. High Alt Med Biol. 2021;16:000-000. https://doi.org/10.1089/ham.2020.0142

[23] Pinilla OCV. Exercise and Training at Altitudes: Physiological Effects and Protocols, Rev Cienc Salud. 2014;12(1):107-122. https://doi.org/10.12804/revsalud12.1.2014.07

[24] Nagashima K, Mack GW, Haskell A, Nishiyasu T, Nadel ER. Mechanism for the Posture-Specific Plasma Volume Increase After a Single Intense Exercise Protocol. J Appl Physiol. 1999;86(3):867-873. https://doi.org/10.1152/jappl.1999.86.3.867

[25] Sawka MN, Convertino VA, Eichner ER, Schneider SM, Young AJ. A Blood Volume: Importance and Adaptations to Exercise Training, Environmental Stresses, and Trauma/Sickness. Med Sci Sports Exerc. 2000;32:332-348. https://doi. org/10.1097/00005768-200002000-00012

[26] Engelen M, Porszasz J, Riley M, Wasserman K, Maehara K, Barstow TJ. Effects of hypoxic hypoxia on O2 uptake and heart rate kinetics during heavy exercise. J Appl Physiol. 1996;81(6):2500-2508. https://doi.org/10.1152/jappl.1996.81.6.2500 
Baltic Journal of Health and Physical Activity 2021; 13 (4): 59-71

Journal of Gdansk University of Physical Education and Sport

e-ISSN 2080-9999 